\title{
Audit \\ Training and supervision of electroconvulsive treatment in a psychiatric training rotation
}

\author{
N. Ramsay Senior Registrar in Psychiatry; and M. A. McPhillips, Registrar in \\ Psychiatry, St Mary Abbots Hospital, Marloes Road London W8 5LQ
}

In 1992, Pippard reported a comprehensive audit of electroconvulsive treatment, (ECT), in two NHS regions. He found deficiencies in most aspects of ECT administration (Pippard, 1992), particularly the training and supervision of the administering doctor, usually an SHO or registrar. Regrettably, the competence of a trainee to administer ECT appeared to have improved but little in the 11 years since the same author first reported on the subject (Pippard \& Ellam, 1981). In particular, it failed to meet the guidelines issued by the Royal College of Psychiatrists in 1989. This was reflected in inadequate administration of ECT as assessed by direct observation and by determining the provision made for teaching and supervision of ECT by senior staff. Pippard concluded that the overall standard of training was inadequate and that "The responsibility for this mediocrity rests squarely on the consultants as a whole and not just on those whose nomination to be in charge appears to absolve the rest ...".

Concern over the above findings led us to audit the level of training and supervision in a large London training rotation.

\section{The study}

We selected six hospitals, each an approved training centre. Of $\mathbf{4 0}$ trainees currently involved in administering ECT, 31 were interviewed by MM using a questionnaire constructed for the purpose. A wide range of experience was covered, ranging from GP trainees on six-month attachments to registrars who had obtained Part II of the MRCPsych. The questionnaire aimed to assess the trainees' actual clinical practice rather than theoretical knowledge.

Areas covered included length of psychiatric training, amount of training in ECT before unsupervised administration, nature of formal and informal teaching, other sources of information, understanding of apparatus, procedure in the event of a failed attempt to induce a fit, understanding of the relationship between convulsion duration and therapeutic effect, and awareness of the Hamilton cuff technique for assessing fit duration. In addition, informal questioning about attitudes to ECT took place.

\section{Findings}

\section{Time in training}

We contacted five GP trainees, (average time in post 4.6 months, range 2 to 6 ), eight SHOs yet to obtain Part I MRCPsych, (average time on training rotation 13.3 months, range 6 to 23), 11 registrars preparing to sit Part II MRCPsych, (average 26.2 months, range 18 to 42), and seven post-membership registrars, (average 43.3 months, range 36 to 54). The duration of training was similar to that of all the trainees on the rotation.

\section{Point in training when ECT commenced}

Almost all of our respondents (93\%) had commenced giving ECT in the first month of their first job in adult psychiatry. There were two who had not given it for two to four months and four who had begun training in mental handicap posts where no ECT was given. Fifteen ( $48 \%$ ) commented that they had given it in the first week.

\section{Amount of training given before giving lists unsupervised}

Seventeen $(55 \%)$ of our sample were given a lecture on the theory of ECT by a senior colleague and 18 $(58 \%)$ had been given a practical demonstration by a senior registrar or consultant. Twelve (39\%) had been given a handout to read. Ten $(32 \%)$ had been instructed by a junior colleague only and one had been shown the electrode positions by the anaesthetist doing the list.

\section{Subsequent training}

It is apparent that once juniors have given a list unsupervised they are regarded as 'trained' as over the ensuing months and years; only three trainees recalled being practically instructed by a senior 
colleague. Nineteen $(61 \%)$ could remember ever having given ECT in the presence of a consultant or senior registrar and in 18 cases this was on the first occasion of giving ECT. Five $(16 \%)$ could recall no formal training other than the advice of junior colleagues and these included an even spread between GP trainees (1), SHOs (1), registrars (2) and one post-membership registrar with four years' experience.

\section{Other sources of education}

Many trainees had made efforts to educate themselves in the field of ECT. Twenty-one $(68 \%)$ had read the report of the ECT Sub-Committee of the Research Committee of the Royal College of Psychiatrists. Twenty-three (74\%) had read textbook accounts or journal reports, or both, or attended Journal Clubs where papers concerning ECT had been presented.

\section{Attitudes to ECT and training}

All those interviewed believed in the usefulness of ECT as a treatment. Several stressed the importance of patient selection. All approved of the use of ECT. Only twenty-four (77\%) regarded themselves as adequately trained in its administration. The remaining seven encompassed a wide spread of seniority including one GP, two SHOs, three registrars and one post-membership registrar. Other than comments on the apparatus in use, trainees commented on poor facilities for patients, poor organisation of ECT lists and varied concerns about the anaesthetist's role.

\section{Arrangements for giving ECT}

Almost without exception, ECT was given twice weekly and bilaterally. No trainee could recall giving unilateral ECT more than once. In all but one hospital ECT was given on a rota system requiring the trainee to give a list once every two to six weeks. In the remaining hospital there were no formal arrangements and ECT was given by the patients' own doctor. In only two hospitals out of six were any of the trainees aware of the existence of a named consultant in charge of ECT as recommended in the guidelines of the Royal College of Psychiatrists (1989).

\section{Apparatus}

Thirteen (42\%) trainees indicated that they did not understand the settings on the machine they were currently using. A variety of machines was in use in the six hospitals and in some two or more types of machine were in routine use. The most common criticism voiced by trainees of any aspect of ECT practice was that the machines varied in each hospital and that they were all old, invoking little confidence in their state of repair.

\section{Settings used}

Questioned about what settings were routinely employed, $21(68 \%)$ stated that they always used the same settings when delivering treatment. This appeared to be by individual choice rather than hospital policy, however, as trainees from the same hospital employed different settings. Only seven $(23 \%)$ reported that they altered the dose of electricity given in a rational way according to previous response. Three trainees reported that they used whatever setting had been employed last time and that they had never actually commenced a course.

\section{Procedure in the event of no fit}

All but one trainee reported that in the event of no seizure at all they would re-stimulate. Only $63 \%$ reported that they would increase the dose of electricity before doing so, the remainder stating that they would use the same settings. All reported that they would discontinue the procedure if a second stimulation failed and four volunteered additional suggestions such as checking the contacts, checking the drug chart for benzodiazepines and discussing the anaesthetic with the anaesthetist.

\section{Seizure duration}

All trainees appeared aware of the need to time seizures but many were uncertain of the reasons for so doing. Asked how long a fit was required to prevent them re-stimulating the patients, a wide spread of answers was obtained. Fourteen (45\%) felt that any fit, however brief, was satisfactory. A further eight $(26 \%)$ felt that less than 15 seconds was adequate, including one GP trainee who felt that seizures over ten seconds were excessive and made a point of reducing the settings to promote briefer seizures. Only nine $(29 \%)$ actively sought seizures in excess of 15 seconds although seven more stated that, although they were aware that longer seizures were probably more therapeutic, they did not make a policy of re-stimulating to obtain seizures of over 15 seconds.

\section{Hamilton cuff method}

Eight $(26 \%)$ trainees reported occasional use of this technique to ascertain seizure length. The remainder had never used it or had never heard of it.

\section{Other observations}

Only one person directly criticised the training they had received, most seeming to feel that they had had the same training as everyone else.

\section{Comments}

The results demonstrate widespread deficiencies in the training of junior psychiatrists to administer 
ECT. If anything, these results are somewhat worse than the observations of Pippard, possibly because of the effect of being observed upon the practitioners.

It is apparent that extremely junior psychiatrists will be required to administer ECT very shortly after taking up post. Up to a third will receive no more instruction than the advice of another junior colleague and this situation is likely to remain unremedied throughout their training. Although a majority will pick up knowledge through journals, textbooks, exam preparation and Journal Clubs, there is scant evidence that this improves technique. There is reason for particular concern in the case of GP trainees who share ECT rotas but do not complete a psychiatric training.

The evidence we present suggests that trainees are strong advocates of the use of ECT and believe it to be effective. Nevertheless, between a fifth and a quarter of those interviewed felt less than competent to give ECT. Ignorance of the apparatus was present in over $\mathbf{4 0} \%$. Regarding actual practice, a minority of trainees alter the dose in a rational manner and over one third respond to a failed stimulation with stimulation at the same settings, whereas increasing the dosage is recommended (Royal College Guidelines, 1989). It is rarer still for trainees to re-stimulate in the event of a brief or partial seizure and all but one considered seizure lengths of less than 25 seconds to be therapeutic, whereas the Royal College guidelines recommend 25 seconds as an "approximate clinical guide". Most trainees are unaware of the Hamilton cuff technique for more accurate timing of seizure length.
The majority of our trainees administered ECT on a rota basis, a practice criticised by Pippard (1992), on the grounds that a trainee rarely sees a patient through an entire course, re-inforcing the "buttonpusher" mentality. However properly ECT may be prescribed, the use of unsupervised and inadequately trained operators must lead to continuing poor practice and reduced therapeutic effectiveness of this powerful treatment.

What of the future? Benbow (1986) has reported on the usefulness of video-taped demonstrations of ECT practice in improving trainees' administration of ECT. Following the report of Pippard (1992), the Royal College of Psychiatrists has commenced an initiative to train consultants and anaesthetists in ECT techniques and theory. Eventually such initiatives will filter down to the training grades. For the moment, our study suggests that training in and supervision of ECT given by junior psychiatrists is at best patchy and, in many instances, seriously deficient.

\section{References}

BENBOW, S. M. (1986) Effect of training on administration of electroconvulsive therapy by junior doctors. Convulsive Therapy, 2, 19-24.

PIPPARD, J. (1992) Audit of electroconvulsive treatment in two NHS regions. British Journal of Psychiatry, 160, $621-637$.

_ \& EllaM, L. (1981) Electroconvulsive treatment in Great Britain. British Journal of Psychiatry, 139, 563-568.

Royal College of Pyschiatrists (1989) The Practical Administration of Electroconvulsive Therapy. London: Gaskell.

\section{Carbamazepine monitoring}

\section{Carol Paton, Staff Pharmacist; and Andrew W. Procter, Senior Lecturer, Bexley Hospital, Bexley, Kent DA5 2BW}

\section{Background of the study}

\section{Rationale for monitoring carbamazepine levels}

Carbamazepine fulfils some of the criteria required in order to render a drug suitable for serum level monitoring in that there is poor inter-individual reproducibility of the serum levels achieved with fixed dosage regimes, interbrand differences in bioavailability are known to occur and serum levels can be raised significantly by several commonly prescribed drugs; dextropropoxyphene (a constituent of coprozamol), erythromycin, isoniazid and cimetidine. 